EXCLUSIVE TO VITAL!

This article is adapted from a chapter of the second edition of Advanced dental nursing edited by Robert Ireland and published by Wiley Blackwell in May 2010 (£29.99)

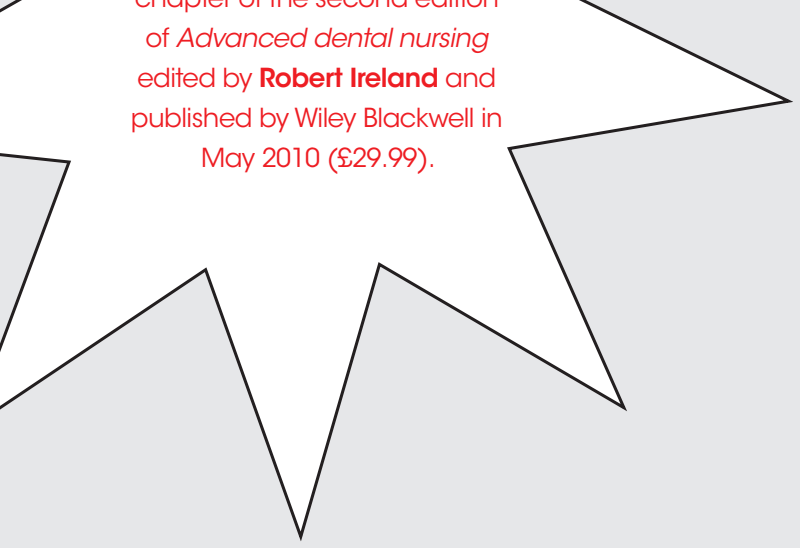

\section{ADVANCED DENTAL NURSING SERIES}

COMMUNICATION

CLINICAL GOVERNANCE

ORAL HEALTH EDUCATION

SPECIAL CARE DENTISTRY

DENTAL ANXIETY

IMPLANT NURSING

ORTHODONTIC NURSING

\title{
Implant nursing
}

\section{By Ulpee Darbar,}

Consultant in

Restorative Dentistry

at Eastman Dental

Hospital and Institute

in London, and Robert

S. Ireland, Associate

Clinical Professor at

Warwick Dentistry, The

University of Warwick

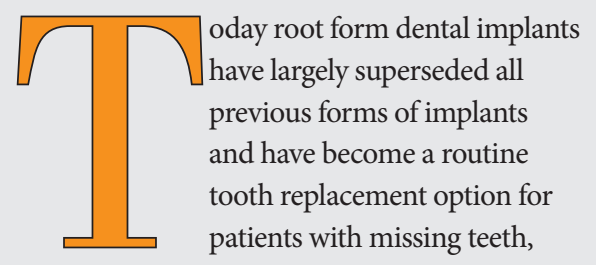

offering patients functional and psychological comfort. However, implant treatment, if not planned and executed appropriately, can also become a traumatic event for the patient. The key aim of implant treatment is to provide the patient with a tooth or teeth that resemble their own, thus improving function, aesthetics and psychological well being. Predictable treatment outcomes with dental implants depends on careful patient selection and treatment planning as well as the integrated working of the whole team which includes the patient, dental nurse, clinician, technician, hygienist and radiographer. The dental nurse's role is vital to ensuring the smooth delivery of the treatment; however, the nurse also plays a pivotal role for the patient who often feels more comfortable approaching the nurse with their concerns. Dental nurses therefore should be aware of their role in the delivery of treatment with dental implants and as a result be aware of the changing concepts and risks associated with such treatment. This article provides an overview of the role of the dental nurse in the delivery of implant treatment to the patient.

Dental nurses and implant treatment The dental nurse plays an essential role throughout the process relating to planning a patient for implant treatment. However, there are three areas where the nurse's involvement is crucial:

- During the planning stage

- During the surgery and prosthetic stages

- After the completion of the treatment.

\section{During the planning stage}

The nurse should have knowledge about dental implants, how they work and the stages of treatment alongside the risks associated with this treatment modality as patients often approach the dental nurse first for any queries they may have about the suggested implant treatment.

Modern day dental implants are devices made from a biocompatible material (usually commercially pure titanium) which is placed within the bone of the jaws (maxilla or mandible), to provide support for a prosthetic reconstruction which could be a single tooth, multiple teeth or all the teeth (eg a denture). Once placed, the implants integrate into the jaw bone, a process called 'osseointegration', which only occurs if a standardised and controlled protocol is followed during the surgical placement of the implants. Osseointegration is a time related phenomenon and its predictability depends on the surface of the implant (also called a fixture), the host bed (environment in which the fixture is placed) and the patient. Previously healing periods of six months were the norm for the integration to occur, however, with changes in the surface topography of the dental implant, this healing period has been reduced with restoration of the fixture with a crown or denture on the same day as the surgery 


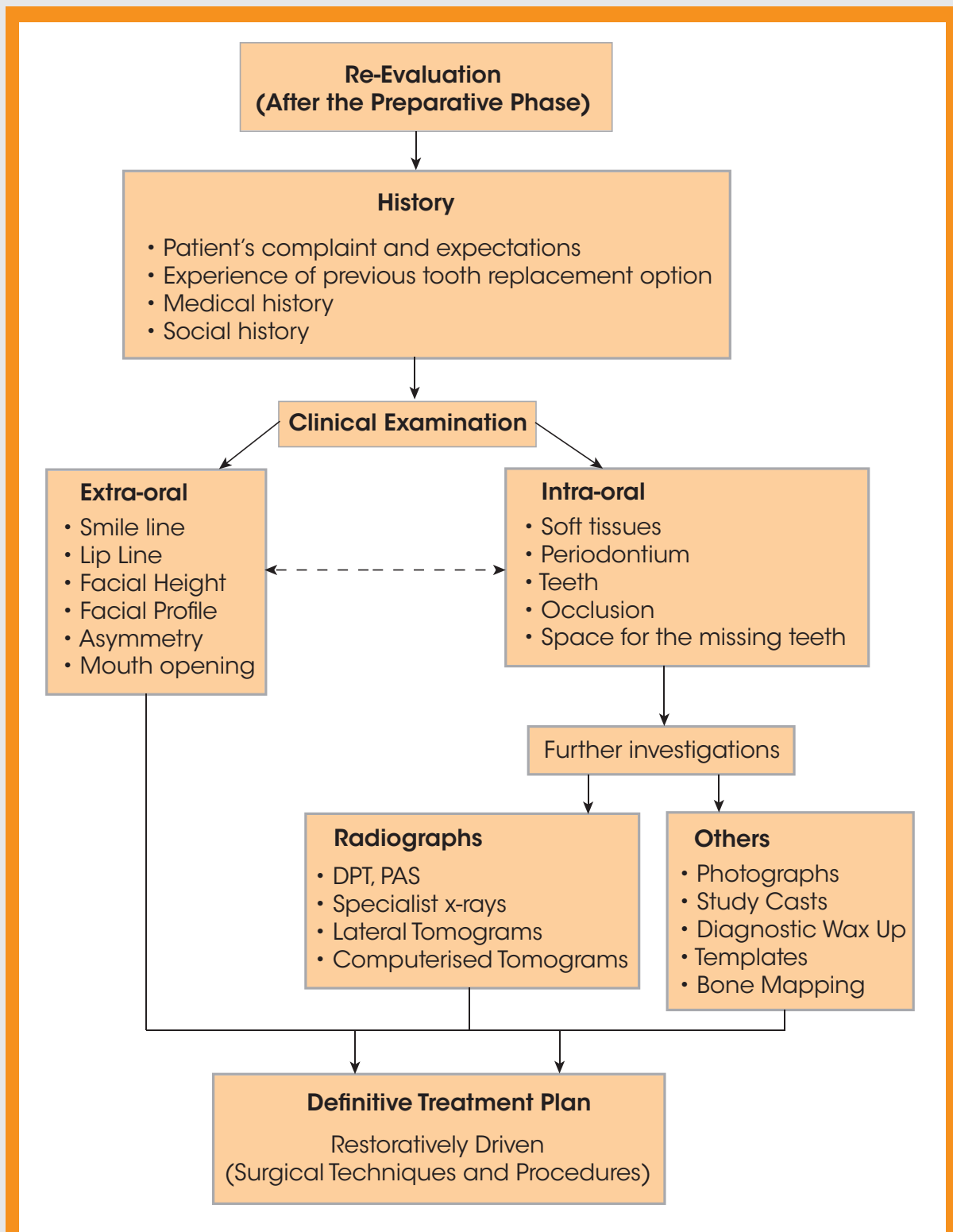

Fig. 1 Steps undertaken during the definitive planning for implant treatment

Table 1 Decision making process - key factors that need to be discussed with the patient

The type of implant retained restoration that will be feasible

The need for bone augmentation either prior to or during the fixture placement

The need for soft tissue augmentation

The type of surgery required and how it would be undertaken

The healing period and the time frame within which the patient will have the final prosthesis

The risks associated with treatment which includes failure of the fixture to integrate into the bone, trauma or damage to any adjacent vital structures

The short and long term complications associated with the treatment

The need for long term maintenance and support to ensure longevity of the restorations.

becoming a reality. For the latter to be feasible it is essential that careful patient selection and planning takes place as immediate restoration is not feasible in all patients and is dependent on the surgical technique and the host site.

The patient assessment usually takes place in
'Dental nurses

ensure the

smooth delivery

of treatment and

play a pivotal role

for the patient

who often feels

more comfortable

approaching the

dental nurse with

their concerns.'

two stages:

- The preoperative planning phase

- The definitive planning phase.

In the preoperative planning phase primary dental problems including concerns with the medical and social history are evaluated and addressed. During this phase the patient's compliance with treatment and their commitment is also evaluated. The dental nurse should be aware of the discussion the dentist has had with the patient during this phase as the patient may often seek clarification about the suggested treatment. At the end of this the definitive planning phase starts.

The definitive planning phase includes a series of steps detailed in Figure 1 that enable a risk assured treatment plan to be drawn up prior to the execution of the implant treatment. The patient remains at the core of this decision making process (see Table 1) and the dental nurse should be aware of what is being planned as they will be responsible for ensuring that all the required work is available in time for the procedure.

The number and site of implants is determined by the type of prosthetic reconstruction planned to address the patient's presenting concerns which may be functional, aesthetic or both. This type of planning is called 'restoratively driven planning' where the position of the final teeth is determined using mock up teeth (called diagnostic wax up) on stone casts that resemble the patient's mouth which are shown to the patient. Aesthetically 
acceptable conventional prostheses can also be used for this purpose. The prosthesis can either be removable or fixed and the choice will depend on the amount of bone and soft tissue lost as well as the patient's wishes. A removable prosthesis is easier to clean and maintain and requires fewer implants to support it whereas a fixed prosthesis cannot be removed by the patient and needs to be designed carefully such that the patient can still access and clean around the implants supporting the prosthesis. This latter type of prosthesis is usually more expensive. A fixed prosthesis can either be screw retained (the crown or bridge is screwed into the implants) or cement retained (the crown or bridge is cemented onto the abutments which are connected to the implants) and the choice between these will be determined by the wax up and the tooth position and profile. Once a decision about the type of prosthesis is made, the surgical planning takes place such that the placement of the implants is undertaken to facilitate the final restoration being in the planned position to maintain the aesthetics and function.

The surgical planning will involve a decision between the staging of the surgical placement (either as 1-stage where the top of the healing cap is visible in the mouth at the end of the surgery or a 2-stage where the healing cap is covered by the gum tissue) and the type of placement. The different types of placement are described in Table 2. At this time during the surgical planning the need for augmenting the jaw bone either at the time of implant placement or before the implant placement will also be made and the choice of material to be used will be determined in conjunction with the patient. Augmentation at the time of surgery is only possible if the implant is firmly stable when screwed into the jaw bone, a phenomenon called 'primary stability'.

The engagement of the patient in this whole process is crucial as it will enable the patient to make an informed decision about their treatment. At the end of the planning phase the implant nurse should be aware of the following:

- The implant system to be used

- The size, number and site in which the implants are to be placed

- The type of surgical placement that is going to be undertaken

- Any surgical guides or devices to be used during the surgery

- Any specialised $\mathrm{x}$-rays that the clinician will want to refer to at the time of surgery

- The need for augmentation and materials needed

- Any specific surgical techniques that the clinician may want to use and the associated equipment needed

\section{Table 2 Types of implant placement as determined by the timing of the extraction}

Standard placement: This was very common in the past. It is used when teeth have been lost for a period of time and the gingivae have completely healed over the site of the missing teeth. The patient may already have a conventional denture or bridge or a space

Delayed placement: This is much more popular today. The teeth are extracted and the gingivae allowed to heal for a period of 6-8 weeks. The implants are then placed after this period

Immediate placement: The teeth are extracted and the implant is placed immediately into the extraction socket. This method is technique sensitive and can cause compromises in the aesthetic region

Teeth in a day: This uses specialised computerised guided stents that are made to fit the patient's mouth. No gingival flaps are raised and the stent is made beforehand aided by specialised $x$-rays.

Table 3 Stages of prosthetic reconstruction

Impression taking (undertaken in the clinic)

Pouring the working cast (undertaken in the laboratory)

Jaw registration (undertaken in the clinic)

Construction of the framework and substructure for the prosthesis (undertaken in the laboratory)

Framework try in (undertaken in the clinic)

Construction of the crowns/bridges/dentures (undertaken in the laboratory)

Try in (undertaken in the clinic)

Fit (undertaken in the clinic.

Table 4 Risks associated with implant treatment

\begin{tabular}{ll}
$\begin{array}{l}\text { During planning } \\
\text { phase }\end{array}$ & $\begin{array}{l}\text { Flare up of infection associated with the teeth adjacent to the } \\
\text { implant site }\end{array}$ \\
& $\begin{array}{l}\text { Fracture of teeth } \\
\text { Trauma to the flap } \\
\text { Excessive bleeding }\end{array}$ \\
$\begin{array}{l}\text { During surgical } \\
\text { placement }\end{array}$ & $\begin{array}{l}\text { Perforation during site preparation } \\
\text { Damage to the nerve }\end{array}$ \\
& $\begin{array}{l}\text { Perforation of the sinus floor } \\
\text { Swelling and bruising }\end{array}$ \\
\hline $\begin{array}{l}\text { Immediately after } \\
\text { the surgery }\end{array}$ & $\begin{array}{l}\text { Infection } \\
\text { Numbness }\end{array}$ \\
\hline $\begin{array}{l}\text { At the prosthetic } \\
\text { connection or after } \\
\text { fit of the prosthesis }\end{array}$ & $\begin{array}{l}\text { Failure of the implant } \\
\text { Loosening or fracture of the screw (abutment or prosthetic) - } \\
\text { Foracture or wear of the bridge or crown }\end{array}$ \\
\hline Inflammation of the gingival tissues around the prosthesis
\end{tabular}


- The components and drilling protocol for the implant system to be used

- The consent documentation that the patient has signed.

It is crucial that the nurse is acquainted and familiarised with the above, especially the particular implant system that is going to be used, since whilst all implant systems follow similar drilling protocols, each has their own specific names for the different components and it is important that the nurse is familiar with these especially if they are using more than one system. A nurse who is forewarned and well prepared is forearmed for those in case' scenarios where if something breaks down during the surgery, there is a fall back plan in place. Additionally the patient may often ask the nurse questions about technical terms which they may not understand.

\section{During the surgery and prosthetic stages}

On the day of the surgery, the implant nurse should be well prepared and know exactly what the clinician has planned to enable appropriate preparation for the surgical procedure in advance. This is important as patients are often nervous on the day of the surgery and will seek comfort and reassurance from the nurse. If the nurse has participated in the planning process they will be better placed to support the clinician and the patient during the course of the surgery. The check list described earlier will help the nurse ensure that any additional equipment and materials needed are available well before the day of surgery especially if they are working alone. The nurse should also be familiar with the concepts of asepsis and antisepsis as these are critical to ensuring that the surgical procedure is undertaken under optimal conditions to achieve the best outcome. The key roles the nurse will have during the surgical procedure will be:

- Setting up and preparing for the surgery

- Ensuring that the required implants and equipment including materials are available

- The stents and other diagnostic aides needed are available

- Assistance during the surgery

- Reassurance to the patient before, during and after the surgery

- Clearing up after the surgery and ensuring that the drills (if they are multiple use) are logged

- Ensure that all the lot number of the implants and materials used are logged in the appropriate places.

The nurse's role in the prosthetic stages is equally important. The prosthesis can be provided immediately after the implant is

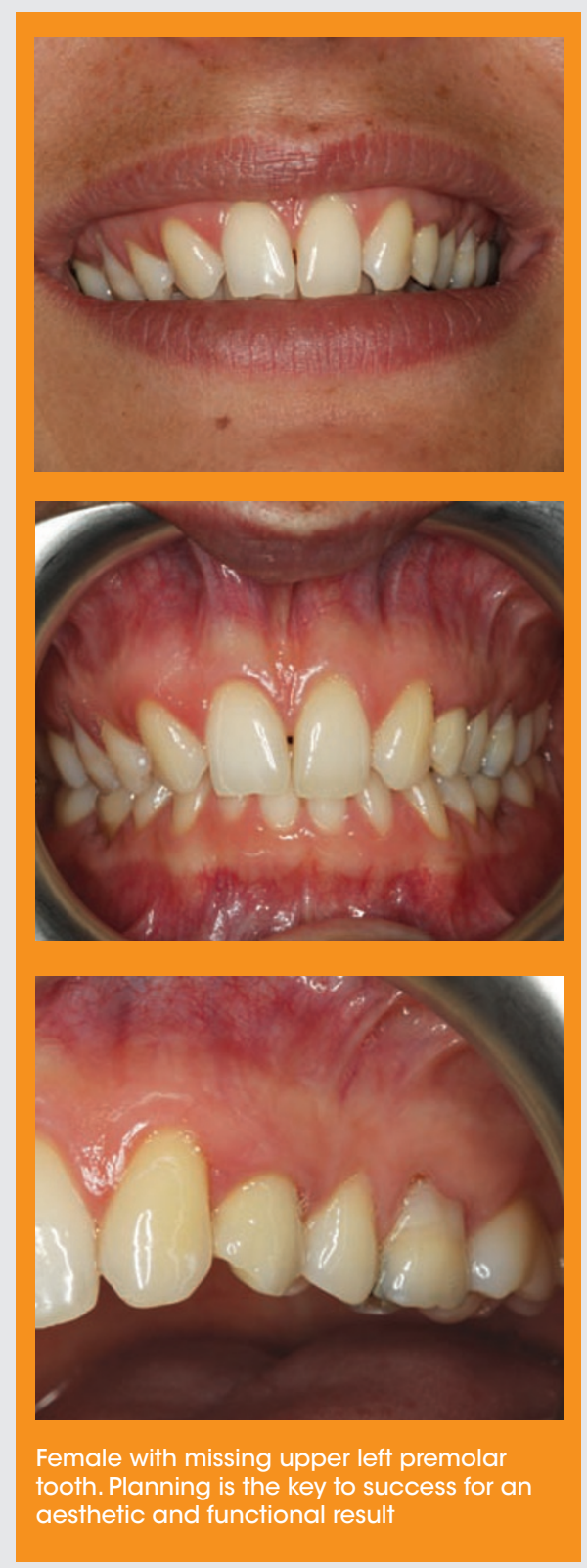

surgically placed (immediate restoration), after 4-8 weeks of implant placement (delayed restoration) or after a period of 4-6 months after implant placement. The time at which the restoration will be provided is dependent on a number of factors, the primary stability and bone quality being the key ones. The nurse has to be aware of when the clinician is going to take the impressions for constructing the prosthesis as they will need to ensure that the correct components and materials needed for taking the impressions are available. Additionally they will have to liaise with the technician about getting the work completed and so should also be familiar with the stages of the prosthetic reconstruction. Table 3 details the main stages involved in construction of a routine implant retained restoration. Patients may often telephone after the surgery because they are worried or have concerns. The nurse should be able to reassure the patient and inform the clinician if needed.
Completion of the treatment

Once the implant retained reconstruction has been provided for the patient, the nurse should ensure that the patient has the correct information needed and details of the treatment provided have been logged. The nurse also has a duty to ensure that the implant stock is maintained and monitored as well as the stock of any other materials and equipment used. The nurse should be aware of the potential complications and risks the patient may suffer both short and long term in the event a patient telephones with a problem. This knowledge will help the nurse prioritise an appointment for the patient especially as they may have taken the call from an anxious patient whom they would be able to reassure.

\section{Risks associated with implant treatment}

Risks can take place at different levels of the delivery of implant treatment to a patient. These can take place during the:

- Planning phase

- Surgical placement and immediately afterwards

- At prosthetic connection and after fit of the prosthesis.

The majority of risks associated with the planning phase can be minimised or avoided with thorough planning and good patient cooperation. The surgical risks can also be minimised with good operative and surgical skills and care during the surgery with the use of suction and flap handling and retraction. Traumatic flap handling can lead to increased postoperative swelling and pain. Table 4 details the different risks that can occur at these stages. The nurse's awareness of these risks will help ensure that they can provide the patient with the best possible care, the correct information and be able to reassure them.

The role of the dental nurse in the successful outcome of implant treatment cannot be underestimated. This is vitally important today especially as all those involved in the delivery of care for patients are responsible and are as much at risk medico-legally as the clinician. To this end the dental nurse must be aware of the importance of data confidentiality, the importance of case record documentation and informed consent. In the event of any concerns the dental nurse should raise these issues with the clinician concerned to ensure that patient safety and care are optimised. Nurses involved in assisting with dental implant nursing should be proactive in the team to ensure that they are able to provide the support and help to the clinician and the patient as is necessitated by their role. 